\title{
Binding affinities of paclitaxel and docetaxel for generic and nanoparticle albumin-bound paclitaxel-derived albumin from human serum
}

\author{
TAKAMICHI SATO, MANAMI OKAZAKI, JUNKO SANO, CHIHIRO KATO, \\ KAZUHISA SHIMIZU and MASAYUKI KITAGAWA
}

Pharmaceutical Research Laboratories, Nippon Kayaku Co., Ltd., Tokyo 115-0042, Japan

Received October 30, 2020; Accepted January 21, 2021

DOI: $10.3892 / b r .2021 .1411$

\begin{abstract}
Nanoparticle albumin-bound (nab)-paclitaxel is a 130-nm formulation containing human serum albumin (HSA). The clinical efficacy of this formulation is considered to depend on its affinity for HSA. The high pressure employed during the manufacture of nab-paclitaxel HSA (nab HSA) may influence its conformation and/or oligomerization, and ultimately its affinity for HSA. Therefore, studies are required to evaluate whether the affinity of paclitaxel for nab HSA is similar to that of generic HSA (control HSA). In the present study, nab HSA was isolated from nab-paclitaxel by gel filtration, and the binding affinities $\left(\mathrm{K}_{\mathrm{D}} \mathrm{s}\right)$ were determined by surface plasmon resonance. Furthermore, the affinity of docetaxel for nab HSA and control HSA was measured, as their binding sites are similar. Paclitaxel showed $\mathrm{K}_{\mathrm{D}} \mathrm{S}$ of $8.93 \pm 8.60$ and $7.39 \pm 5.81 \mu \mathrm{M}$ for nab HSA and control HSA, respectively, whereas the corresponding $\mathrm{K}_{\mathrm{D}} \mathrm{S}$ for docetaxel were $44.3 \pm 9.50$ and $55.9 \pm 2.28 \mu \mathrm{M}$, respectively. This suggests that the paclitaxel binding site was not modified during the nab-paclitaxel manufacturing process. Additionally, nab HSA likely does not affect paclitaxel and blood HSA binding, as evidenced by the similar affinities of paclitaxel and docetaxel for nab HSA and control HSA. In conclusion, the binding affinities of paclitaxel and docetaxel for nab HSA and control HSA were found to be comparable. Additionally, the manufacturing process did not influence the paclitaxel binding affinity for nab HSA. These results also suggest that nab HSA may not affect the clinical effectiveness of nab-paclitaxel.
\end{abstract}

Correspondence to: Dr Takamichi Sato, Pharmaceutical Research Laboratories, Nippon Kayaku Co., Ltd., 3-31-12 Shimo, Kita-ku, Tokyo 115-0042, Japan

E-mail: takamichi.sato@nipponkayaku.co.jp

Key words: binding affinity, docetaxel, human serum albumin, paclitaxel, surface plasmon resonance

\section{Introduction}

Nanoparticle albumin-bound (nab)-paclitaxel is a nanoparticle formulation of human serum albumin (HSA) that is $130 \mathrm{~nm}$ in size (1-3). Taxol, another paclitaxel formulation, forms micelles in the blood after injection, prolonging the time of increased paclitaxel concentrations in the blood. This leads to high hematological toxicity $(2,3)$. In contrast, nab-paclitaxel nanoparticles collapse immediately after injection. Therefore, when the incorporated paclitaxel is released into the bloodstream, it binds to HSA $(2,3)$, and thus the increased blood paclitaxel concentration is not prolonged. Indeed, the recommended therapeutic dose of nab-paclitaxel is higher than that of Taxol (4).

The intratumoral paclitaxel levels were found to be elevated in an in vivo xenograft model following nab-paclitaxel treatment relative to treatment with a Cremophor-based paclitaxel formulation (1) that is similar to Taxol. Albumin is transcytosed through endothelial cells via the albumin receptor gp60, and tumor cells take up albumin as an energy source (5). The higher therapeutic efficacy of nab-paclitaxel relative to Taxol (4) is considered to result from the reduced hematological toxicity and increased accumulation of paclitaxel in tumors mediated by albumin receptors. Therefore, the binding affinity between blood HSA and paclitaxel is an important factor for assessing its efficacy.

High pressure is used to manufacture nab-paclitaxel (2), which may affect the conformation and oligomerization of nab-paclitaxel HSA (nab HSA), as HSA oligomers have different biochemical characteristics from those of monomeric HSA (6-8). If the manufacturing process of nab-paclitaxel influences oligomer formation and/or the native HSA structure, the binding affinity of paclitaxel for nab HSA may differ from that of generic HSA, possibly reducing the efficacy of nab-paclitaxel in the clinical setting. As indicated above, nab-paclitaxel has superior therapeutic efficacy to Taxol (4), suggesting that nab HSA has similar biochemical characteristics to generic HSA and that binding affinities of both HSAs for paclitaxel are comparable.

To confirm the hypothesis that nab HSA is similar to generic HSA, the binding affinities of paclitaxel for nab HSA and generic HSA (control HSA) were compared. In addition, 
the affinities of docetaxel for nab HSA and control HSA were determined, as their binding sites are similar (9).

\section{Materials and methods}

Chemical reagents and proteins. Docetaxel was purchased from Tokyo Chemical Industry. Paclitaxel was purchased from IndenaSpA. HSA and ubiquitin were purchased from Sigma-Aldrich; Merck KGaA. Unless indicated otherwise, all other chemicals were of analytical reagent grade and purchased from commercial sources.

Depletion of paclitaxel from nab-paclitaxel HSA. An aliquot of nab-paclitaxel (7.5 mg; Celgene Corporation) was dissolved in $2.5 \mathrm{ml}$ PBS (pH 7.4; Thermo Fisher Scientific, Inc.). The solution was centrifuged at $20,000 \mathrm{x}$ g for $15 \mathrm{~min}$ at $4^{\circ} \mathrm{C}$, and the supernatant was collected (HSA fraction). The treatment was repeated with PD10 (GE Healthcare) three times to deplete the paclitaxel. The PD10-treated fraction was dialyzed against PBS three times with a Slide-A-Lyzer dialysis cassette (MW cutoff, $3 \mathrm{kDa}$; Thermo Fisher Scientific, Inc.). The protein concentration in the dialyzed fraction was adjusted to $1 \mathrm{mg} / \mathrm{ml}$ with PBS. The protein concentration was determined using a BCA Protein assay kit (Thermo Fisher Scientific, Inc.).

Paclitaxel quantification. For paclitaxel quantification, ((-)-(1S,2S,3R,4S,5R,7S,8S,10R,13S)-4,10-diacetoxy-2 -benzoyloxy-5,20-epoxy-1,7-dihydroxy-9-oxotax-11-en13-yl( $2 R, 3 S)$-3-benzoylamino-2-hydroxy-3-benzylpropionate) was used as an internal standard (IS). To each $100 \mu \mathrm{l}$ sample, $150 \mu$ lacetonitrile/ethanol solution (2:1) containing $3.3 \mu \mathrm{g} / \mathrm{ml}$ IS was added. The mixture was filtered using a GL ChromatoDisc (diameter $0.45 \mu \mathrm{m}$, GL Science). The reverse-phase-HPLC system LC-20AB prominence series (Shimadzu Corp.) was used. Chromatographic separation was performed using a Wakopak Navi C30 (5 $\mu \mathrm{m}$ particle, 2.0x150 mm; FUJIFILM Wako Pure Chemical) at a column temperature of $40^{\circ} \mathrm{C}$. The mobile phase consisted of $0.1 \%$ aqueous phosphoric acid (A) and acetonitrile (B). Isocratic elution with $47 \%$ (B) was performed for $50 \mathrm{~min}$ at a flow rate of $0.25 \mathrm{ml} / \mathrm{min}$ whilst monitoring the UV absorption at $254 \mathrm{~nm}$. Paclitaxel and IS were eluted at 10.8 and $15.6 \mathrm{~min}$, respectively.

Native PAGE and Coomassie Brilliant blue (CBB) staining. Native PAGE was performed as described previously with slight modifications (10). Briefly, $10 \mu 12 \mathrm{X}$ concentrated sample buffer ( $20 \%$ glycerol and $0.05 \%$ bromophenol blue) was added to $10 \mu 11 \mathrm{mg} / \mathrm{ml}$ HSA solution. The mixture was loaded onto a 5-20\% SuperSep Ace precast gel (FUJIFILM Wako Pure Chemical) and electrophoresed in $3.0 \mathrm{~g} / 1$ Tris(hydroxymethyl) aminomethane and $14.4 \mathrm{~g} / 1$ glycine running buffer. The gel was stained with CBB Stain One Super at $20-25^{\circ} \mathrm{C}$ for $60 \mathrm{~min}$ (Nacalai Tesque).

Binding affinity measurements. Surface plasmon resonance (SPR) analysis was performed using a Biacore X100 (GE Healthcare), with binding affinity determined by multiple-cycle analysis, as described previously $(11,12)$. Briefly, nab HSA or control HSA was immobilized on a CM7 sensor chip with an Amine Coupling kit (GE Healthcare). The target immobilization level was set to 24,000 RU. During preliminary experiments, it was determined that ubiquitin immobilization in the reference cells reduced non-specific binding of paclitaxel (data not shown). Therefore, ubiquitin was immobilized on a reference cell with the Amine Coupling kit by the time and flow method (contact time $420 \mathrm{sec}$ ). Paclitaxel solutions at concentrations of $0.625,1.25$, 2.5, 5.0 and $10 \mu \mathrm{M}$ in $0.5 \%$ DMSO with $4 \mu \mathrm{g} / \mathrm{ml}$ carboxymethyl dextran (MW 10 kDa, Tokyo Chemical Industry) and buffered using $50 \mathrm{mM}$ Tris-HCl buffer to $\mathrm{pH} 7.4$ (Sigma-Aldrich; Merck $\mathrm{KGaA}$ ) were prepared. Each paclitaxel solution was added to the sensor chip at a flow rate of $30 \mu \mathrm{l} / \mathrm{min}$ for $30 \mathrm{sec}$ followed by addition of washing buffer [0.5\% DMSO- $4 \mu \mathrm{g} / \mathrm{ml}$ carboxymethyl dextran (MW $10 \mathrm{kDa})-50 \mathrm{mM}$ Tris-HCl buffer (pH 7.4)] at a flow rate of $30 \mu \mathrm{l} / \mathrm{min}$ for $60 \mathrm{sec}$.

For docetaxel analysis, a CM5 sensor chip (GE Healthcare) was used. The target immobilization levels of nab HSA and control HSA were set to 10,000 RU. As a reference, ubiquitin was immobilized with the Amine Coupling kit using a method similar to that described above. Docetaxel solutions at concentrations of $3.125,6.25,12.5,25,50$ and $100 \mu \mathrm{M}$ in $0.1 \%$ DMSO-0.05\% polyethylene glycol (MW $20 \mathrm{kDa}$; FUJIFILM Wako Pure Chemical) $50 \mathrm{mM}$ Tris-HCl buffer (pH 7.4) were prepared. Each docetaxel solution was added to the sensor chip at a flow rate of $30 \mu \mathrm{l} / \mathrm{min}$ for $30 \mathrm{sec}$ followed by the addition of washing buffer [0.1\% DMSO-0.05\% polyethylene glycol-(MW $20 \mathrm{kDa})-50 \mathrm{mM}$ Tris- $\mathrm{HCl}$ buffer (pH 7.4)] at a flow rate of $30 \mu \mathrm{l} / \mathrm{min}$ for $30 \mathrm{sec}$.

The SPR sensorgram was globally fitted using a 1:1 binding model. $\mathrm{K}_{\mathrm{D}}$ values were calculated using Biacore X100 Evaluation Software version 2.0.1 in affinity mode (GE Healthcare). All SPR experiments were repeated at least three times. Data are presented as the mean \pm standard deviation.

\section{Results}

Characterization of nab HSA. To evaluate whether the paclitaxel binding affinity of nab HSA was similar to that of the control generic albumin, nab HSA was isolated from nab-paclitaxel. The results of isolation of nab HSA and a typical HPLC chromatogram are shown in Table I and Fig. 1A-C, respectively. The results indicated that paclitaxel was successfully depleted by repeated PD-10 treatment. The lowest paclitaxel concentration used in our calibration curve for HPLC analysis was $0.32 \mu \mathrm{M}$, and there was no detectable paclitaxel signal in the fraction treated three times with PD-10 (Fig. 1C), indicating that the remaining paclitaxel concentration was $<0.32 \mu \mathrm{M}$. Additionally, it has been reported that the $\mathrm{K}_{\mathrm{D}}$ values of paclitaxel-HSA binding are $0.42-69.9 \mu \mathrm{M}(10-12)$, indicating that the remaining paclitaxel concentration shown in Table I was lower than the lowest reported $K_{D}$ value. Thus, the isolated nab HSA was suitable for the binding affinity experiments.

Next, whether the albumin isolation steps affected oligomer formation was evaluated. Fig. 2 shows that the amount of oligomer in the isolated nab HSA was similar to that in the parental pretreatment nab HSA $(7,13)$. Thus, the isolation steps showed a limited influence on the oligomer formation of nab HSA.

Paclitaxel and docetaxel binding to nab HSA and control HSA. SPR analysis was analyzed to confirm that the paclitaxel binding affinity of nab HSA was similar to that of nab HSA. 
Table I. Summary of nab HSA purification.

\begin{tabular}{lcccc}
\hline & \multicolumn{2}{c}{ HSA } & \multicolumn{2}{c}{ Paclitaxel } \\
\cline { 2 - 3 } \cline { 5 - 5 } Treatment & HSA, mg & Yield, $\%$ & Paclitaxel, $\mu \mathrm{g} / \mathrm{ml}$ & Yield, $\%$ \\
\hline Pre treatment & 7.5 & 100 & 179.4 & 100.0 \\
Supernatant & 6.9 & 82.1 & 56.8 & 31.6 \\
Precipitate & 0.6 & 92.8 & 192.2 & 64.3 \\
PD10, 1st & 7 & 67 & 2.3 & 1.8 \\
PD10, 2nd & 5 & 50.8 & ND & ND \\
PD10, 3rd & 3.8 & & ND & ND \\
\hline
\end{tabular}

HSA, human serum albumin; ND, not detected.

A

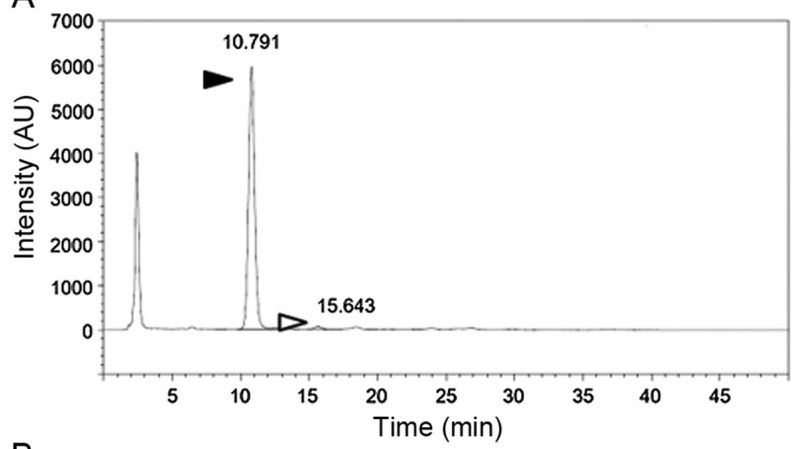

B

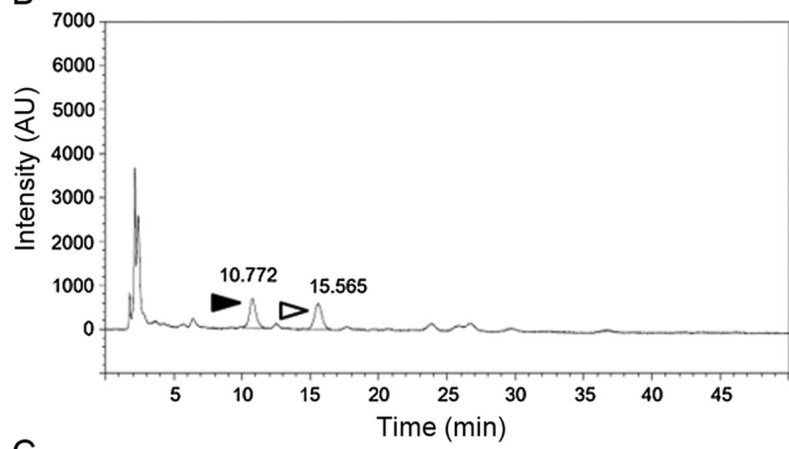

C

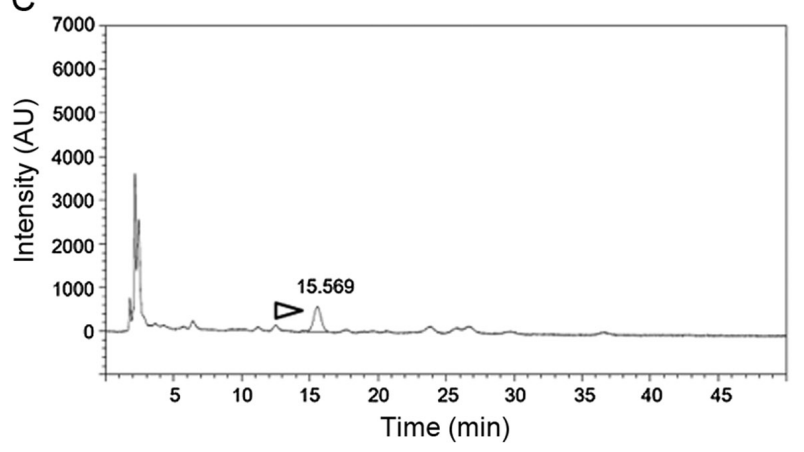

Figure 1. Representative elution profiles of paclitaxel. Concentrations of (A) pre-treatment sample, (B) sample treated with PD10 once (PD10 1st), and $(\mathrm{C})$ sample treated with PD10 three times (PD10 3rd) were analyzed by reverse-phase-HPLC. Paclitaxel and paclitaxel internal standards are indicated with black and white arrows, respectively. AU, arbitrary units.

Representative sensorgrams are shown in Fig. 3A and B. The $\mathrm{K}_{\mathrm{D}}$ values for the binding of paclitaxel to nab HSA and control HSA were similar $(8.93 \pm 8.60$ and $7.39 \pm 5.81 \mu \mathrm{M}$, respectively).

\section{3}

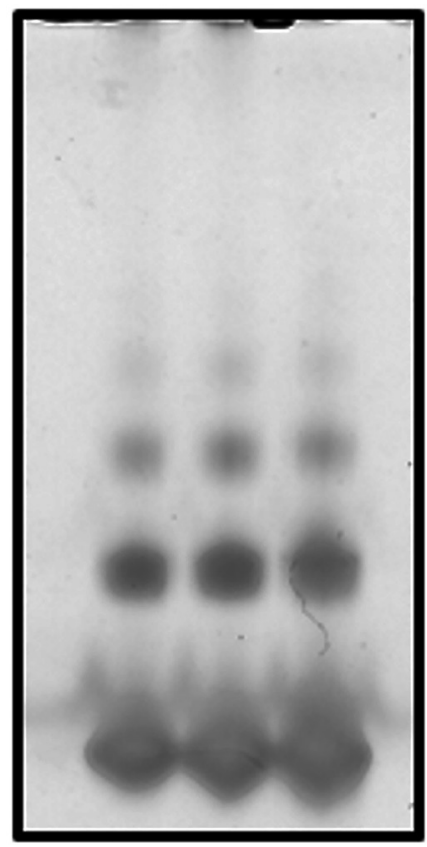

Figure 2. Native-PAGE of nab HSA and control HSA. Oligomer formation rates of pre nab HSA (pretreatment nab HSA), dialyzed nab HSA (dialyzed nab HSA after the purification), and control HSA were analyzed by native-PAGE followed by Coomassie Brilliant blue staining. Lane 1, pretreatment of nab HSA; Lane 2, dialyzed nab HSA; Lane 3, control HSA. HSA, human serum albumin; nab, nanoparticle albumin-bound.

Next, the $\mathrm{K}_{\mathrm{D}} \mathrm{s}$ were determined for the binding of docetaxel to both HSAs, as its binding site on HSA is similar to that of paclitaxel (9). Representative sensorgrams are shown in Fig. $4 \mathrm{~A}$ and B. The $\mathrm{K}_{\mathrm{D}}$ for nab HSA was $44.3 \pm 9.50 \mu \mathrm{M}$, whereas that for control HSA was $55.9 \pm 2.28 \mu \mathrm{M}$, indicating that the docetaxel binding affinities of both HSAs are comparable.

\section{Discussion}

In the present study, SPR experiments were used to confirm the hypothesis that nab HSA is characteristically similar to control generic HSA. The $\mathrm{K}_{\mathrm{D}} \mathrm{S}$ of nab HSA and control HSA for paclitaxel were $8.93 \pm 8.60$ and $7.39 \pm 5.81 \mu \mathrm{M}$, respectively. The affinity of paclitaxel-HSA binding has not been 

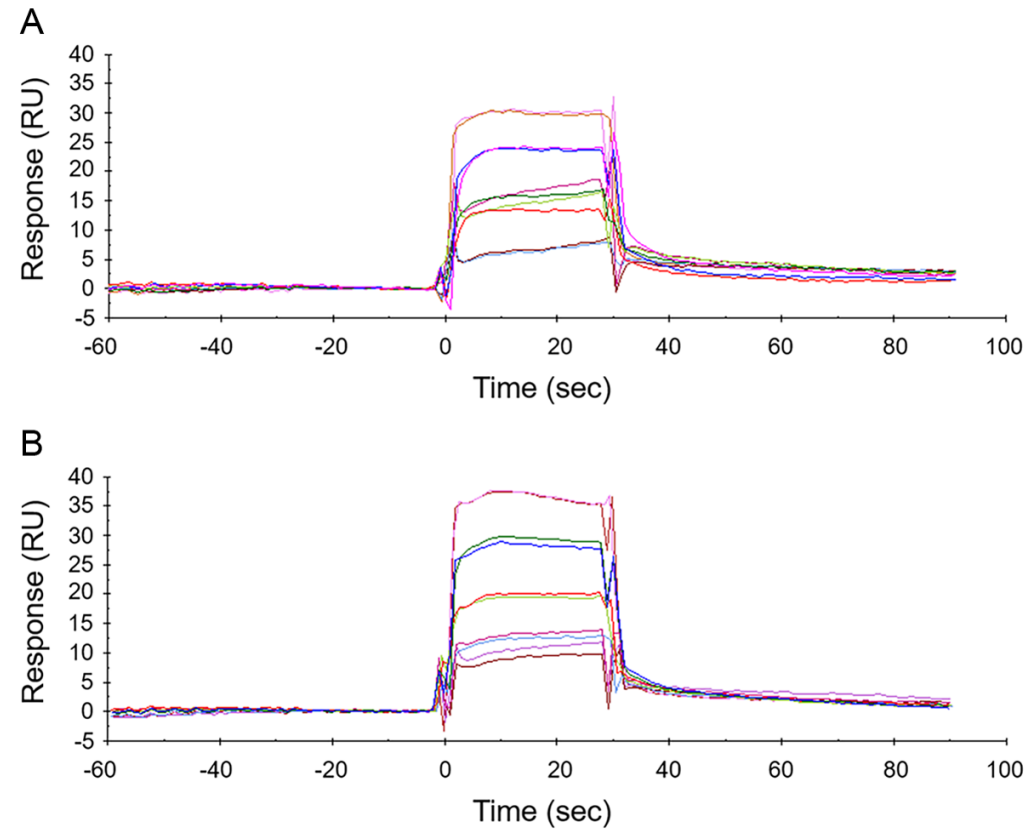

Figure 3. Surface plasmon resonance measurements of binding affinities of paclitaxel for nab HSA and control HSA. Representative sensorgrams. Multiple concentrations of paclitaxel solutions $(0.625,1.25,2.5,5.0$ and $10 \mu \mathrm{M})$ were sequentially added to a (A) nab HSA or (B) control HSA immobilized sensor chip. All experiments were repeated at least three times. HSA, human serum albumin; nab, nanoparticle albumin-bound; RU, resonance units.
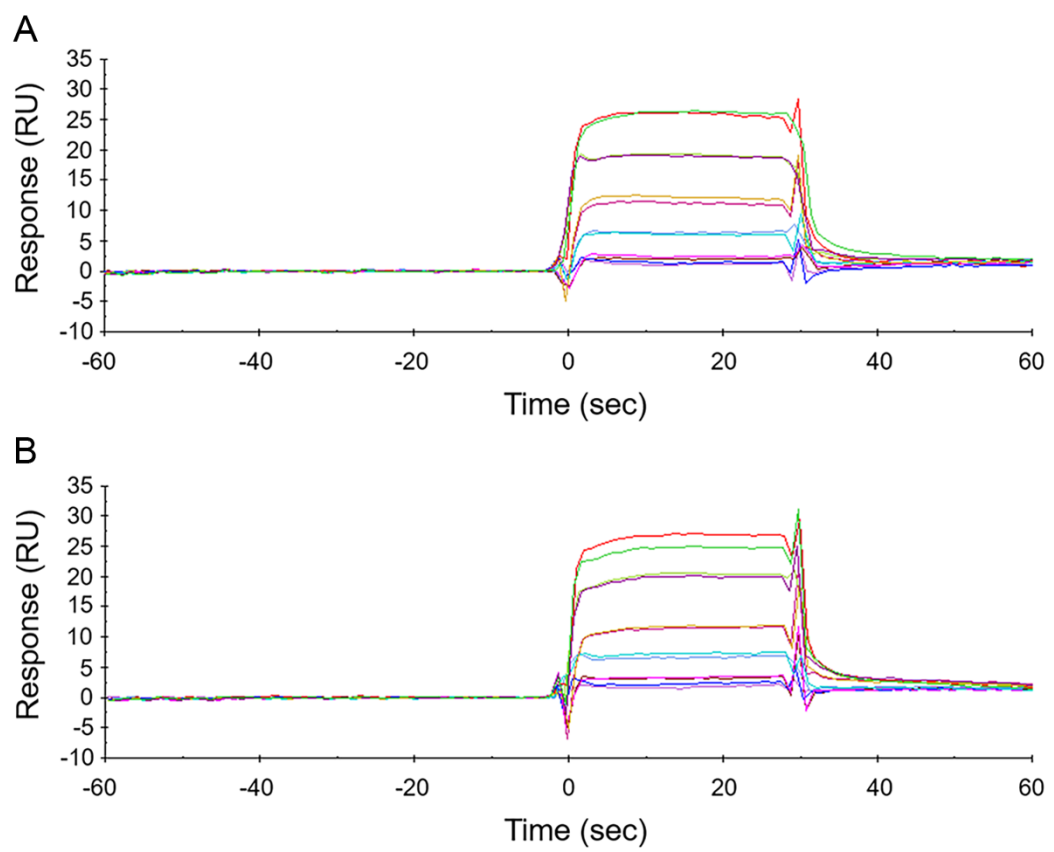

Figure 4. Representative sensorgrams of surface plasmon resonance analysis of docetaxel-nab HSA and control HSA binding. Multiple concentrations of docetaxel $(3.125,6.25,12.5,25,50$ and $100 \mu \mathrm{M})$ were sequentially added to a (A) nab HSA or (B) control HSA immobilized sensor chip. All experiments were repeated at least three times. HSA, human serum albumin; nab, nanoparticle albumin-bound; RU, resonance units.

examined previously by SPR, to the best of our knowledge. The $\mathrm{K}_{\mathrm{D}}$ values of paclitaxel-HSA binding determined by other methods were 0.42-69.9 $\mu \mathrm{M}$ (10-12), consistent with the results of the present study. Additionally, the docetaxel binding affinities for nab HSA and control HSA were determined as the binding region is similar between paclitaxel and docetaxel. The $\mathrm{K}_{\mathrm{D}} \mathrm{S}$ of docetaxel for nab HSA and control HSA were $44.3 \pm 9.50$ and $55.9 \pm 2.28 \mu \mathrm{M}$, respectively, whereas the reported docetaxel $\mathrm{K}_{\mathrm{D}}$ determined by SPR was $199 \mu \mathrm{M}$ (14), showing a discrepancy with the docetaxel-HSA $\mathrm{K}_{\mathrm{D}} \mathrm{s}$ measured in the present study. Paal et al (9) reported the paclitaxel $\mathrm{K}_{\mathrm{D}}$ calculated in a docking study was 80 -fold lower than the experimentally determined $\mathrm{K}_{\mathrm{D}}$. They concluded that this difference was caused by variations in experimental conditions, which may also be the case in the present study.

Fatty acids can bind to HSA and modulate PTX-HSA binding (15), suggesting that contamination by fatty acids affects drug-HSA binding affinities. To address this issue, fatty 
acid-free and highly purified HSA was used as the control HSA. Additionally, pharmaceutical grade nab-paclitaxel purchased from Celgene was used. Regulatory authorities strictly ensure that pharmaceutical products are free from impurities in accordance with ICH Q3 (ich.org/page/quality-guidelines). Therefore, the results of the present study were not influenced by residual impurities.

There are several water-insoluble anti-cancer agents, such as rapamycin and docetaxel. It is suggested that this nab technology is useful for solubilizing these water-insoluble agents. Of interest, nab-rapamycin is under clinical investigation for use as a treatment for sarcomas (16), and Khodaei et al reported that rapamycin may primarily bind to site I on HSA via a probe displacement study (17). As indicated above, nab HSA is characteristically similar to control generic HSA, suggesting that nab HSA does not influence nab-rapamycin efficacy in the clinical setting.

In conclusion, the binding affinities of paclitaxel and docetaxel for nab HSA and control HSA were found to be comparable. Additionally, the manufacturing process did not influence the paclitaxel binding affinity for nab HSA. It is well-established that nab-paclitaxel is more effective than Taxol. This higher therapeutic efficacy of nab-paclitaxel than that of Taxol is considered to result from the reduced hematological toxicity and increased accumulation of paclitaxel in tumors mediated by albumin receptors (4). Therefore, the results of the present study also suggest that nab HSA does not affect the clinical effectiveness of nab-paclitaxel.

\section{Acknowledgements}

The authors would like to thank Dr Takeshi Onda, Dr Shouichi Ohno and Ms. Sayaka Yako (Nippon Kayaku Co., Ltd.) for providing fruitful discussions and technical support.

\section{Funding}

No funding was received.

\section{Availability of data and materials}

The datasets and materials used during the present study are available from the corresponding author upon reasonable request.

\section{Authors' contributions}

KS and MK designed the study. TS, MO, JS and CK designed the study, performed the experiments and wrote the manuscript. TS, KS and MK reviewed and edited the manuscript. All authors have read and approved the final manuscript.

\section{Ethics approval and consent to participate}

Not applicable.

\section{Patient consent for publication}

Not applicable.

\section{Competing interests}

The authors declare that they have no competing interests.

\section{References}

1. Desai N, Trieu V, Yao Z, Louie L, Ci S, Yang A, Tao C, De T, Beals B, Dykes D, et al: Increased antitumor activity, intratumor paclitaxel concentrations, and endothelial cell transport of cremophor-free, albumin-bound paclitaxel, ABI-007, compared with cremophor-based paclitaxel. Clin Cancer Res 12: 1317-1324, 2006.

2. Yardley DA: nab-Paclitaxel mechanisms of action and delivery J Control Release 170: 365-372, 2013.

3. Chen N, Brachmann C, Liu X, Pierce DW, Dey J, Kerwin WS, $\mathrm{Li} \mathrm{Y}$, Zhou S, Hou S, Carleton M, et al: Albumin-bound nanoparticle (nab) paclitaxel exhibits enhanced paclitaxel tissue distribution and tumor penetration. Cancer Chemother Pharmacol 76: 699-712, 2015.

4. Untch M, Jackisch C, Schneeweiss A, Conrad B, Aktas B, Denkert C, Eidtmann H, Wiebringhaus H, Kümmel S, Hilfrich J,etal;German BreastGroup(GBG); Arbeitsgemeinschaft Gynäkologische Onkologie-Breast (AGO-B) Investigators: Nab-paclitaxel versus solvent-based paclitaxel in neoadjuvant chemotherapy for early breast cancer (GeparSepto-GBG 69): A randomised, phase 3 trial. Lancet Oncol 17: 345-356, 2016.

5. Merlot AM, Kalinowski DS and Richardson DR: Unraveling the mysteries of serum albumin-more than just a serum protein. Front Physiol 5: 299, 2014.

6. Sollenne NP, Wu HL and Means GE: Disruption of the tryptophan binding site in the human serum albumin dimer. Arch Biochem Biophys 207: 264-269, 1981.

7. Nakano NI, Shimamori Y and Yamaguchi S: Binding capacities of human serum albumin monomer and dimer by continuous frontal affinity chromatography. J Chromatogr A 237: 225-232, 1982.

8. Matsushita S, Chuang VT, Kanazawa M, Tanase S, Kawai K, Maruyama T, Suenaga A and Otagiri M: Recombinant human serum albumin dimer has high blood circulation activity and low vascular permeability in comparison with native human serum albumin. Pharm Res 23: 882-891, 2006.

9. Paal K, Shkarupin A and Beckford L: Paclitaxel binding to human serum albumin - automated docking studies. Bioorg Med Chem 15: 1323-1329, 2007.

10. Paál K, Müller J and Hegedûs L: High affinity binding of paclitaxel to human serum albumin. Eur J Biochem 268: 2187-2191, 2001

11. Purcell M, Neault JF and Tajmir-Riahi HA: Interaction of taxol with human serum albumin. Biochim Biophys Acta 1478: 61-68, 2000.

12. Amani N, Saberi MR and Chamani JK: Investigation by fluorescence spectroscopy, resonance rayleigh scattering and zeta potential approaches of the separate and simultaneous binding effect of Paclitaxel and estradiol with human serum albumin. Protein Pept Lett 18: 935-951, 2011.

13. Atmeh RF and Shabsoug B: Detection and semiquantitation of albumin forms in fresh human plasma separated on gradient polyacrylamide gel by means of electroblotting on agarose gel matrix. Electrophoresis 18: 2055-2058, 1997.

14. Cimitan S, Lindgren MT, Bertucci C and Danielson UH: Early absorption and distribution analysis of antitumor and anti-AIDS drugs: Lipid membrane and plasma protein interactions. J Med Chem 48: 3536-3546, 2005.

15. Paal K and Shkarupin A: Paclitaxel binding to the fatty acid-induced conformation of human serum albumin - automated docking studies. Bioorg Med Chem 15: 7568-7575, 2007.

16. Gordon EM, Chua-Alcala VS, Kim K, Baby R, Angel N, Quon D, Wong S and Chawla SP: A phase I/II investigation of nivolumab and ABI-009 (nab-sirolimus) in advanced undifferentiated pleomorphic sarcoma (UPS), liposarcoma (LPS), chondrosarcoma (CS), osteosarcoma (OS), and Ewing sarcoma: Preliminary efficacy and safety results. J Clin Oncol 37 (Suppl 15): 11057, 2019.

17. Khodaei A, Bolandnazar S, Valizadeh H, Hasani L and Zakeri-Milani P: Interactions between sirolimus and anti-inflammatory drugs: Competitive binding for human serum albumin. Adv Pharm Bull 6: 227-233, 2016. 